\title{
Elastic oscillations of water column in the 2003 Tokachi-oki tsunami source: in-situ measurements and 3-D numerical modelling
}

\author{
M. A. Nosov and S. V. Kolesov \\ M. V. Lomonosov Moscow State University, Faculty of Physics, Moscow, Russia \\ Received: 5 October 2006 - Revised: 15 January 2007 - Accepted: 24 March 2007 - Published: 2 April 2007
}

\begin{abstract}
During the 2003 Tokachi-Oki tsunamigenic earthquake the real-time JAMSTEC observatory obtained records which provided a unique opportunity to have a look deep inside the tsunami source. Considering water column as a compressible medium we processed the bottom pressure records in order to estimate amplitude, duration and velocity of bottom displacement. Spectral analysis of the records revealed a clear manifestation of the low-frequency elastic oscillations of water column. We also presented 3-D finite-difference numerical model developed in the framework of linear potential theory of ideal compressible fluid to better understand dynamical processes in the tsunami source. The model reproduces position of the main spectral maximum rather correctly. However, due to neglecting of crust elasticity and to lack of exact knowledge of spatiotemporal laws of bottom motion, there is an essential difference between in-situ observed and computed spectra.
\end{abstract}

\section{Introduction}

In recent years, tsunami modelling (Titov et al., 2005; Kowalik et al., 2005), except very few studies (Panza et al., 2000; Ohmachi et al., 2001), has been performed in the framework of incompressible fluid theory. The role of water compressibility in the tsunami evolution was discussed early by Miyoshi (1954), Sells (1965) and Kajiura (1970). Later, we showed theoretically that water elasticity is significant only at the stage of tsunami generation by an earthquake, while tsunami propagation and onshore wave run-up can be described as incompressible fluid motion (Nosov, 1999, 2000).

The main difference in the behavior of a compressible ocean, as compared to an incompressible model medium, is in the formation of elastic oscillations of the wa-

Correspondence to: M. A. Nosov

(nosov@ phys.msu.ru) ter column. In case of a horizontal (absolutely rigid) bottom, the oscillations are characterized by a discrete set of normal frequencies: $v_{k}=c \quad(1+2 k) / 4 H$, where $k=0,1,2, \ldots, c$ is the sound speed in water, $H$ is the oceanic depth. Typical value of the normal frequency is $v_{0} \sim 0.1 \mathrm{~Hz}$ $(H=4000 \mathrm{~m}, c=1500 \mathrm{~m} / \mathrm{s})$.

Actual tsunami sources cover a region with a complex bathymetry. At a certain point of the source, the first to be formed are elastic oscillations with normal frequencies determined by the ocean depth at this point. Later, the spectrum of elastic oscillations can be enriched with high frequencies due to waves that have propagated from the neighboring shallow regions. Owing to the existence of the cut-off frequency (Tolstoy and Clay, 1987), low-frequency oscillations formed in the neighboring deep regions do not propagate upslope. Being manifested only at sufficiently large depths (in the open ocean) these oscillations are not observed near the shore. However, strong elastic oscillations can contribute to the tsunami wave by means of nonlinear mechanisms (Novikova and Ostrovsky, 1982; Nosov and Skachko, 2001; Nosov and Kolesov, 2005).

It is worth noting the differences between the T-phase and the oscillations that are considered in this work. T-phase is a series of seismic waves of relatively high frequency $(>2 \mathrm{~Hz})$ channeled in the ocean water column by SOFAR (Okal et al., 2003). T-phase propagates for a rather long distance from earthquake epicenter, whereas the low-frequency oscillations remain mostly in the epicenter area gradually losing their energy due to the refraction in bottom.

In order to monitor seismicity, tsunamis and other geophysical phenomena in the southern Kurile subduction zone, in July 1999, Japan Agency for Marine-Earth Science and Technology (JAMSTEC, http://www.jamstec.go.jp/) deployed a real-time observatory at the continental slope southeast of Hokkaido (Hirata et al., 2002). The observatory successfully recorded the Tokachi-Oki 2003 earthquake which was the strongest seismic event of the year (Watanabe et

Published by Copernicus GmbH on behalf of the European Geosciences Union. 
al, 2004); the earthquake generated a tsunami with a maximum wave height of 4 meters along the southeastern coast of Hokkaido. This event has been discussed in many studies in respect of either the earthquake or the tsunami source (Yamanaka and Kikuchi, 2003; Miyazaki et al., 2004; Yagi, 2004; Hirata et al., 2004; Tanioka et al., 2004; Mikada et al., 2006).

The records obtained during the Tokachi-Oki 2003 event provide a unique opportunity to verify and to revise, if necessary, the state-of-the-art view on processes in tsunami source. In particular, a clear manifestation of the low-frequency elastic oscillations of water column (normal frequency $v_{0}$ ) has been detected in bottom pressure records (Nosov et al., 2005).

In the present study, we provide further analysis of the data recorded by JAMSTEC bottom pressure sensors during the Tokachi-Oki 2003 tsunamigenic earthquake. We also present a 3-D numerical model developed in the framework of linear potential theory of ideal compressible fluid to better understand dynamical processes in the tsunami source.

\section{Mathematical model}

Let us restrict our approach to a model of an absolutely rigid bottom. This assumption is based on an essential difference which exists in acoustic stiffness of water and bottom. In the region under consideration, P-wave velocity is $V_{p}=6500 \mathrm{~m} / \mathrm{s}$ and density is $\rho_{b}=2780 \mathrm{~kg} / \mathrm{m}^{3}$ (for depth more than $20 \mathrm{~km} V_{p}=7800 \mathrm{~m} / \mathrm{s}$ and $\rho_{b}=3300 \mathrm{~kg} / \mathrm{m}^{3}$ ), whereas for water layer the sound velocity is $c=1500 \mathrm{~m} / \mathrm{s}$ and density is $\rho=1000 \mathrm{~kg} / \mathrm{m}^{3}$ (Yamanaka and Kikuchi, 2003). We want to stress here that finiteness of bottom elasticity does not influence the set of normal frequencies for the water layer; it just leads to a gradual damping of hydroacoustic waves due to their refraction in the bottom. The exponential decay time for energy of hydroacoustic waves (normal incidence) can be estimated as follows (Nosov, 2000):

$\tau_{s}=\frac{H}{c} \frac{(1+B)^{2}}{2 B}$,

where $B=\rho_{b} V_{p} / \rho c(B \approx 12-17)$. It is worth reminding that the decay time for wave amplitude is two times longer. In reality, due to an arbitrary angle of incidence and coupling between elastic oscillations of water and bottom, the observed decay time should exceed the value given by Eq. (1). For the maximum depth of the region $H=7500 \mathrm{~m}$ the decay time can reach $\tau_{s} \sim 50 \mathrm{~s}$. Thus, the elastic oscillations of water layer can be observed at least during a few minutes after bottom earthquake.

In this study, we do not intend to simulate the bottom pressure records performed by JAMSTEC exactly. We are merely trying to simulate the formation of some features of frequency spectra of the records. This is why we do not need to use a realistic time-spatial history of co-seismic bottom deformation. Anyway, realistic permanent vertical deformation is taken into account (Mikada et al., 2006).

Let us consider a layer of an ideal compressible homogeneous fluid in the field of gravity. The layer is bounded by a free surface above and by an absolutely rigid bottom below. The origin of the Cartesian coordinate system $O x y z$ is located at the unperturbed free surface, and the $O z$-axis is oriented vertically upward. We assume the calculation domain to be small enough to neglect the sphericity of the Earth. The bottom position is set by function

$z_{b}(x, y, t)=-H(x, y)+\eta(x, y) \varphi(t)$,

where $H(x, y)$ is the oceanic depth; $\eta(x, y)$ is the vertical permanent co-seismic bottom displacement; and $\varphi(t)$ is the displacement time-history function. To find acoustic and gravitational waves generated by vertical bottom displacement of small amplitude $(|\eta \varphi|<<H)$ the following linear problem for the current velocity potential $F(x, y, z, t)$ is solved:

$\frac{\partial^{2} F}{\partial t^{2}}-c^{2} \Delta F=0$,

$\frac{\partial^{2} F}{\partial t^{2}}=-g \frac{\partial F}{\partial z}, \quad z=0$,

$\frac{\partial F}{\partial \boldsymbol{n}}=n_{z} \eta \frac{\partial \varphi}{\partial t}, \quad z=-H(x, y)$,

where $\boldsymbol{n} \equiv\left(n_{x}, n_{y}, n_{z}\right)$ is the unit vector normal to the bottom surface; $g$ is the acceleration of gravity. The function for vertical bottom displacement velocity is specified in the following form:

$\frac{\partial \varphi}{\partial t}=0.5\left[1-\cos \left(\frac{2 \pi t}{\tau}\right)\right][\theta(t)-\theta(t-\tau)]$,

where $\tau$ is the bottom displacement duration; $\theta$ is the Heviside step function. The non-reflective boundary conditions for acoustic waves are specified at open-sea boundaries. Along the coastline the normal velocity is set to zero. The dynamic pressure $p$ and the fluid surface displacement $\xi$ are expressed via the current velocity potential by the following formulae:

$p=-\rho \frac{\partial F}{\partial t}, \quad \xi=-\left.\frac{1}{g} \frac{\partial F}{\partial t}\right|_{z=0}$.

The traditional explicit Finite Difference scheme (rectangular grid) is used for Eqs. (3-7). The condition for stability of the scheme is Courant criterion $\Delta t<\min (\Delta x, \Delta y, \Delta z) / c$, where $\Delta t$ is time increment, $\Delta x, \Delta y$ and $\Delta z$ are space increments. During the numerical experiments, the time increment was computed as follows: $\Delta t=0.5 \Delta z / c$.

The normal derivative in Eq. (5) is calculated in the following way. The number of nodes in vertical direction $N$ at a given point $(x, y)$ is calculated as rounded ratio $H(x, y) / \Delta z$. 
The space increment $\Delta z$ is chosen so that for each neighboring bottom boundary nodes the difference between the number of nodes in vertical direction can be 0 or \pm 1 . Each bottom boundary node $N(x, y)$ has four closest neighbors: $N(x \pm \Delta x, y)$ and $N(x, y \pm \Delta y)$. These five points determine four planes (triangles) which cross each other at the bottom node $N(x, y)$. Averaging normals to these four planes gives the desired normal at the given bottom node. Intersection of the normal and of the plane $z=[N(x, y)-2] \Delta z$ determines a quasi-internal point $(x+\delta x, y+\delta y)$. Value of the current velocity potential at the quasi-internal point $F^{\text {int }}$ is calculated as linear interpolation from the neighboring nodes which are situated on the plane $z=[N(x, y)-2] \Delta z$; all of them are surely internal points. It is worth noting that similar nodes of plane $z=[N(x, y)-1] \Delta z$ can be either internal or bottom boundary points. Finally, the normal derivative is approximated by the following FD formula:

$$
\frac{\partial F}{\partial \boldsymbol{n}} \approx \frac{F^{\mathrm{int}}-F^{\mathrm{bot}}}{\sqrt{\delta x^{2}+\delta y^{2}+4 \Delta z^{2}}},
$$

where $F^{\text {bot }}$ is the current velocity potential value at a given bottom point.

The computational domain is shown in Fig. 1. It extends from $142.6 \mathrm{E}$ to $145.6 \mathrm{E}$ in longitude and from $40.5 \mathrm{~N}$ to $43 \mathrm{~N}$ in latitude (approx. $280 \times 250 \mathrm{~km}$ ). The $500 \mathrm{~m}$ gridded bathymetric data set of Japan Oceanographic Data Center (JODC, http://www.jodc.go.jp/) was used to build the computational grid. In calculations the following values of space increments were used: $\Delta x \approx \Delta y \approx 500 \mathrm{~m}, \Delta z \approx 140 \mathrm{~m}$. The total number of the grid points was close to $10^{7}$. The time increment was $\Delta t \approx 0.05 s$. In the numerical experiments the duration $\tau$ was varied within the range from 2 to $20 \mathrm{~s}$. The highest frequency we try to reproduce in our modeling is of $0.5 \mathrm{~Hz}$. This frequency corresponds to the shortest hydroacoustic wave length of $3 \mathrm{~km}$. The space increments and the time increment are small enough to perform adequate modeling.

The numerical model was carefully tested on the exact analytical solution of the same problem for the case of constant depth (Nosov, 2000).

\section{Discussion of results}

Figure 2 depicts an example of the 3-D numerical simulation performed at bottom displacement duration $\tau=5 \mathrm{~s}$. The snapshot of water surface disturbance corresponds to the time of $60 \mathrm{~s}$ after the bottom displacement beginning. The surface below shows the bottom topography in the computational domain. Considering rather smooth spatial distribution of the bottom displacement, one can expect nearly the same form of the water surface elevation. Nevertheless, a complicated fast oscillating wave structure can be seen on the water surface. The structure is a surface manifestation of the elastic

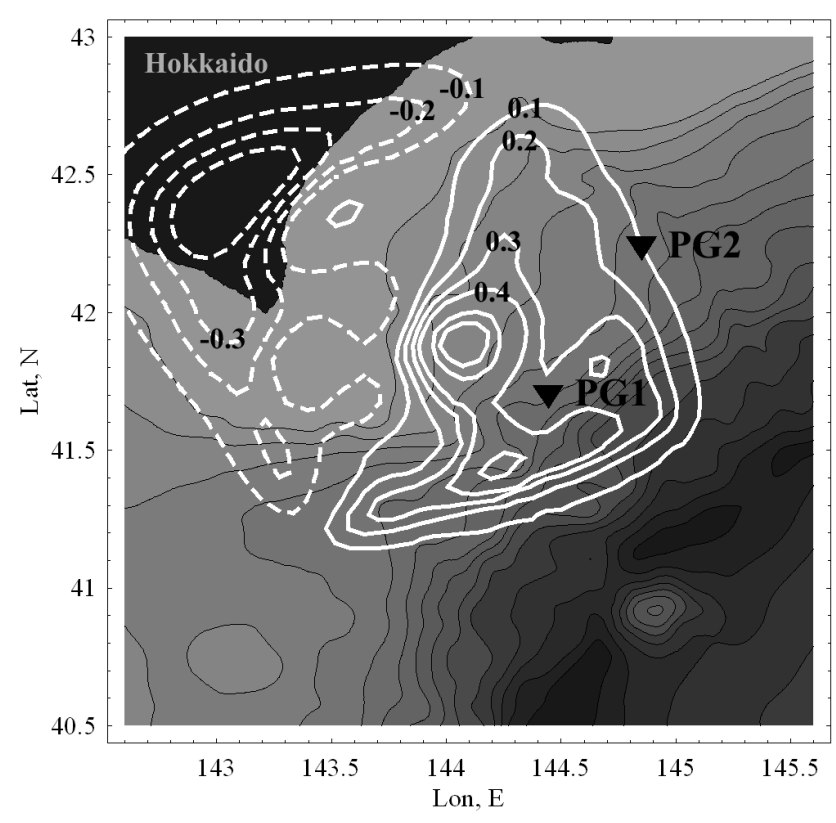

Fig. 1. Computational domain. Isobaths are shown at 500-m intervals. Location of the bottom pressure sensors (black triangles). The vertical co-seismic bottom deformation is shown by solid (uplift) and dashed (subsidence) white contours at 0.1-m intervals.

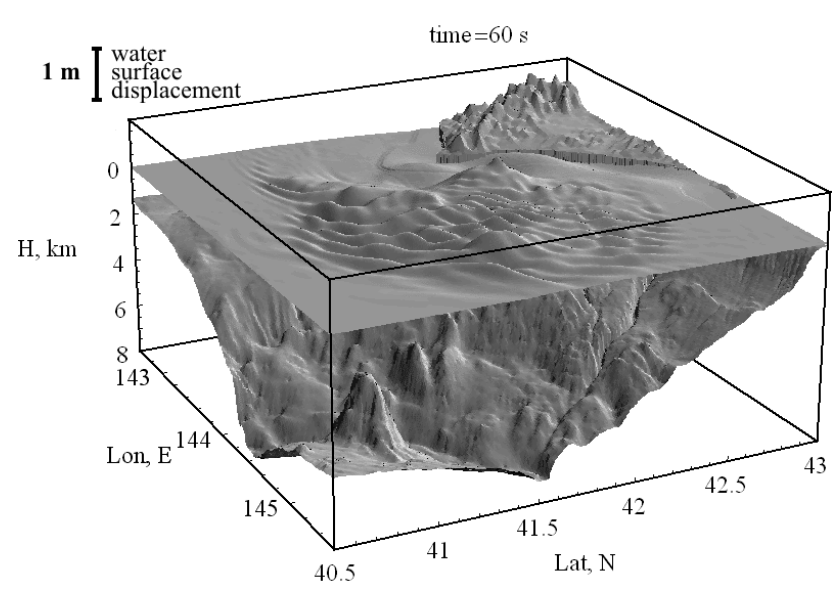

Fig. 2. Computed surface manifestation of the elastic oscillations of the water layer generated by bottom displacement and bottom topography (surface below).

oscillations of the water layer. It is seen from Fig. 4 that double amplitude of the wave structure is comparable with vertical bottom displacement $\left(\eta_{\max }=0.79 \mathrm{~m}, \eta_{\min }=-0.55 \mathrm{~m}\right)$. So the first stage of the tsunami life can be disguised by elastic oscillations. Actually, the amplitude depends on bottom displacement duration. In case under consideration, at short duration $\tau=2 \mathrm{~s}$ the amplitude reaches up to $1.5 \mathrm{~m}$; whereas for longer durations it is noticeably smaller $(5 \mathrm{~s}-1.25 \mathrm{~m}$; $8 \mathrm{~s}-$ $1.1 \mathrm{~m})$. 


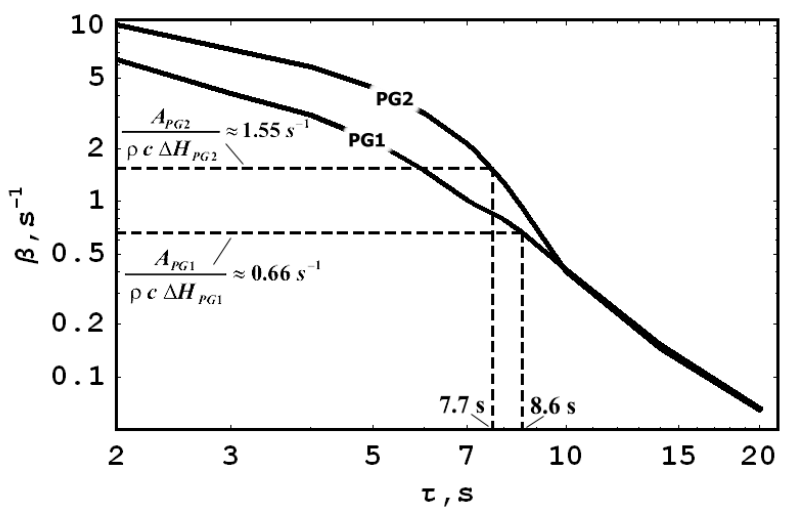

Fig. 3. Function $\beta_{P G i}(\tau)$ calculated from numerical experiments. Scheme of estimation of bottom displacement duration.
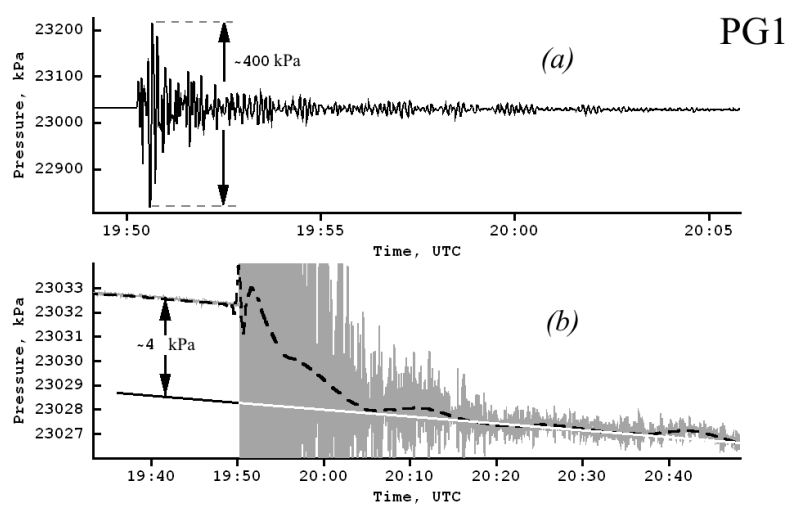

Fig. 4. The bottom pressure recorded by the PG1 sensor at the Tokachi-oki 2003 tsunami source: (a) original data; (b) data plotted on a larger scale. Arrows show (a) the double amplitude and (b) the permanent pressure changes after the earthquake. Dashed line stands for the low-frequency component of the signal $(0-0.02 \mathrm{~Hz})$.

As it has been shown theoretically (Nosov, 2000) for the case of flat horizontal bottom, the wave amplitude in the structure, being a nonmonotone function of the duration, tends to decrease as $\tau^{-1}$. In case of rapid bottom motion $\tau<<H / c$ the amplitude is several times larger in the magnitude than the bottom displacement and, consequently, larger than the initial water surface elevation. Results of numerical modeling performed on a realistic complex bathymetry and theoretical solution of the idealized problem are certainly not completely equal.

Considering simultaneous bottom displacement specified by Eq. (6) synthetic pressure variations at the positions of PG sensors were calculated. First, we analyzed how the double amplitude of the synthetic signals $A=p_{\max }-p_{\min }$ depends on maximum bottom displacement velocity

$U=2 \eta / \tau$.

The following simple relation is fulfilled in an idealized onedimensional case:

$A=\rho c U$.
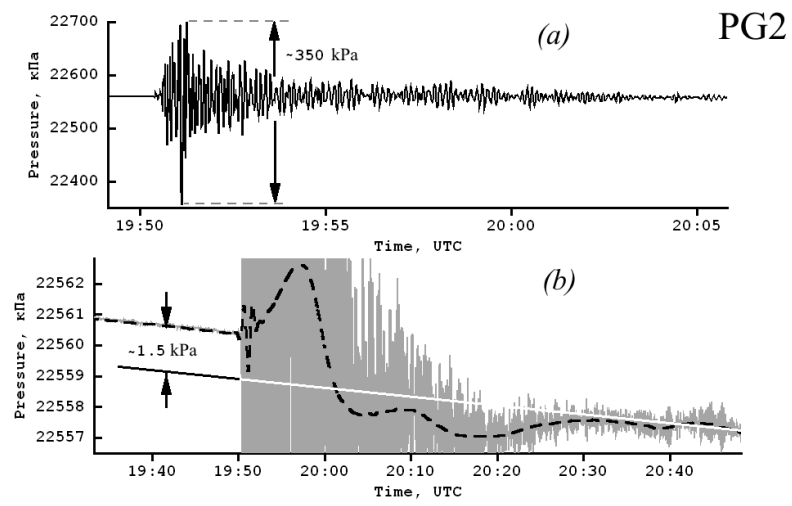

Fig. 5. The bottom pressure recorded by the PG2 sensor at the Tokachi-oki 2003 tsunami source: (a) original data; (b) data plotted on a larger scale. Arrows show (a) the double amplitude and (b) the permanent pressure changes after the earthquake. Dashed line stands for the low-frequency component of the signal $(0-0.02 \mathrm{~Hz})$.

Realistic 3-D modeling involves a complex bathymetry as well as complex distribution of bottom displacement. Due to interference of hydroacoustic waves, the amplitude $A$ at a given point depends on both point coordinates $(x, y)$ and the duration $\tau$. Thus, for each PG sensor there is a specific relation $A_{P G i}=\alpha_{P G i}(\tau) \rho c U_{P G i}$. Taking into account Eq. (9) the relation is written in the following form:

$A_{P G i}=\beta_{P G i}(\tau) \rho c \eta_{P G i}$.

The function $\beta_{P G i}(\tau)$ is a response function that represents an integral influence of the bottom bathymetry and the spatial distribution of the permanent bottom displacement on the double amplitude of synthetic pressure variations at a given point. The dependence $\beta_{P G i}(\tau)$ plotted on Fig. 3 was tabulated from series of numerical simulations.

In what follows the dependence is used to estimate bottom displacement duration during the Tokachi-Oki 2003 earthquake. Such method is surely more advanced than the simplest one based on direct using of relations (9) and (10). However, due to neglecting of crust elasticity and to lack of exact knowledge of spatiotemporal laws of bottom motion in numerical modeling, it is still rough estimation rather than exact calculation.

The time-history of bottom pressure variations recorded by JAMSTEC sensors PG1 and PG2 is shown in Fig. 4a and Fig. 5a. The double amplitude of the variations reached $A_{P G 1} \approx 400 \mathrm{kPa}$ and $A_{P G 2} \approx 350 \mathrm{kPa}$.

A small permanent decrease of bottom pressure took place after the earthquake: $\Delta p_{P G 1} \approx 4 \mathrm{kPa}, \Delta p_{P G 2} \approx 1.5 \mathrm{kPa}$ (Watanabe et al., 2004; Nosov et al., 2005; Mikada et al., 2006); it can be clearly seen on larger scale plots which are shown on Fig. 4b and Fig. 5b. These small permanent changes of pressure resulted from the residual coseismic bottom uplift $\Delta H=\Delta p / \rho g\left(\Delta H_{P G 1} \approx 0.4 \mathrm{~m}\right.$ and $\Delta H_{P G 2} \approx$ $0.15 \mathrm{~m}$ ). It should be noted that coseismic uplift $\eta_{P G i}$ 
estimated from the slip by Mikada et al. (2006) is slightly smaller than the measured values $\Delta H_{P G i}\left(\eta_{P G 1}=0.304 \mathrm{~m}\right.$, $\eta_{P G 2}=0.103 \mathrm{~m}$ ).

Considering the uplift $\Delta H_{P G i}$ and the double amplitude $A_{P G i}$, we calculate a value of the function $\beta_{P G i}(\tau)$ from the Eq. (9). Finally, in the way shown on Fig. 3, we obtain estimation of the bottom displacement duration or rise time $\left(\tau_{P G 1} \approx 8.6 \mathrm{~s}\right.$ and $\left.\tau_{P G 2} \approx 7.7 \mathrm{~s}\right)$. A rather close value of the rise time for M8 earthquake ( $\sim 6 \mathrm{~s})$ can be obtained from empirical laws (see e.g. Kanamori and Anderson, 1975).

According to the Harvard CMT Catalog, the half duration of the process in the Tokachi-Oki 2003 earthquake source was $\tau_{E Q}=33.5 \mathrm{~s}$. The close value of the total source duration (50 s) was reported by Yagi (2003). We note that $\tau_{P G i}<\tau_{E Q}$. This is not surprising, because $\tau_{E Q}$ is associated with the duration of the rupture formation process whereas $\tau$ is the duration of bottom deformation at the given point. Thus, the bottom deformation did not occur smoothly and simultaneously in the entire active region. On the one hand, a "travellingwave" bottom displacement might occur. Further, a rather slow bottom deformation during the period $\sim \tau_{E Q}$ might be accompanied by a relatively fast bottom "trembling" providing a resonant pumping of energy to the elastic oscillations of water column.

Considering Eq. (9), from $\tau_{P G i}$ and $A_{P G i}$ we estimate the vertical bottom displacement velocity. The estimate yields reasonable values for the vertical bottom velocity at the positions of pressure sensors: $U_{P G 1} \approx 0.093 \mathrm{~m} / \mathrm{s}$ and $U_{P G 2} \approx 0.039 \mathrm{~m} / \mathrm{s}$.

As mentioned above, the ocean depth is responsible for the set of normal frequencies. It means that the frequency spectrum of elastic oscillations of the water layer at a given point of a tsunami source is inseparably connected with the bottom topography in the vicinity of the point. Generally speaking, the time-spatial history of bottom displacement and the bottom elasticity are also capable of affecting the spectrum; however, in our opinion, the resonant response of water layer and therefore the role of bathymetry, are still more important. This is why in numerical modeling, we use reliable JODC bathymetric data, whereas for the bottom displacement we merely assume that a simultaneous bottom uplift (subsidence) occurs during a certain period $\tau$.

Below we discuss features of frequency spectra of bottom pressure variations that have been recorded in-situ by JAMSTEC sensors (PG1, PG2) and that have been calculated using the 3-D model.

The spectra of the in-situ records are shown in Fig. 6a. The $1000 \mathrm{~s}$ long time series were used to calculate the spectra. Initial data were reduced to the zero level by means of subtracting the linear trend. We note that only the lowest normal frequency can be observed in the spectra. All other normal frequencies exceed the Nyquist frequency $(0.5 \mathrm{~Hz})$.

It is seen from Fig. 6a that both spectra are characterized by clearly manifested main maxima. The maxima for sensors PG1 and PG2 are located within the ranges 0.14-
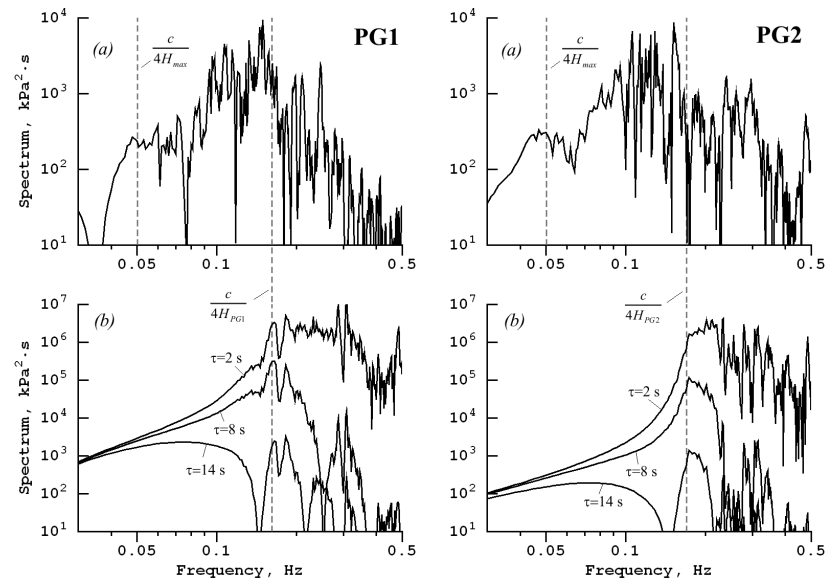

Fig. 6. Frequency spectra of bottom pressure variations: (a) in-situ recorded by JAMSTEC sensors, (b) calculated using 3-D numerical model at different bottom displacement durations. Gray dashed lines stand for the positions of normal frequencies at a certain depth.

$0.15 \mathrm{~Hz}$ and $0.15-0.16 \mathrm{~Hz}$ respectively. It is very important that the location of maxima on the frequency scale is different. Hence, they are related not to the spectral characteristics of the seismic source but rather to the resonance response of the compressible water column at the minimal normal frequency. Sensor PG1 is located in a deeper place. Therefore, it corresponds to a lower normal frequency. In order to estimate the value of normal frequency $v_{0}=c / 4 H$, we simply use the sound velocity in water $c=1500 \mathrm{~m} / \mathrm{s}$ and ocean depth at the location of pressure sensor $\left(H_{P G 1}=2322 \mathrm{~m}\right.$ and $H_{P G 2}=2216 \mathrm{~m}$ ). The result of the estimation is shown in Figure 6 by gray dashed lines. One can see that the pressure variations actually occur at frequencies typical of the elastic oscillations of the water column, but the main maxima are slightly shifted to the left of the theoretical predictions. The shift of the maxima is explained by the fact that the acoustic basement is located under a thick sediment layer (Nosov et al., 2005). Another remarkable feature of the spectra is the existence of secondary rather weak maxima at frequency of $v \approx 0.05 \mathrm{~Hz}$ (dashed gray line on the left). Due to the cutoff frequency the elastic oscillations of water column are trapped by a local depression of the bottom. The southern Kuril Trench is such a depression to trap the oscillations. We note that the maximum depth of the southern Kuril Trench $H_{\max } \approx 7500 \mathrm{~m}$ corresponds well to the observed frequency of secondary maxima, i.e. $v=c / 4 H_{\max }$. The actual bottom rocks are not absolutely rigid, therefore, such low-frequency oscillations reach sensors PG1 and PG2 not as hydroacoustic waves, the cut-off frequency effect does not allow it, but as seismic waves. The bottom elasticity is also responsible for the high level of energy in the range nearly $0.1 \mathrm{~Hz}$.

Finally, we consider spectra of synthetic bottom pressure variations calculated for the locations of sensors PG1 and PG2 using the 3-D numerical model. The spectra of the 
synthetic signals calculated for different bottom displacement durations are shown in Fig. 6b. As it has been expected, maxima of the spectra appear at the theoretically predicted positions which are depicted by gray dashed line $\left(c / 4 H_{P G i}\right)$. Spectral level strongly depends on the bottom displacement duration. Nevertheless, some peaks of the synthetic spectra exist at any value of bottom duration. This remarkable feature confirms our suggestion regarding the leading role of bottom topography in formation of elastic oscillations spectrum. At first sight, it makes sense to compare in-situ and synthetic spectra in order to reveal similar peaks. However, at the present stage, such comparison can be misleading. Out of other parameters, the normal frequency is pretty sensitive to the elastic features of sedimentary layers which are not taken into account in the present study.

As it was estimated above, the bottom displacement duration for the Tokachi-Oki 2003 earthquake was about $8 \mathrm{~s}$. It is seen from Fig. 6 that the synthetic spectra calculated at $\tau=8 \mathrm{~s}$ has essentially higher level than in situ measured one. Such difference can be explained as a consequence of neglecting the bottom elasticity. Rather energetic spectral components of the frequency range $\sim 0.1 \mathrm{~Hz}$ contribute to the measured signals. However, these components can not be reproduced in our modeling. Neglecting the bottom elasticity also leads to a certain overestimation of high-frequency signals $v>c / 4 H_{P G i}$. Propagating down the slope from the shallow water region these signals contribute to the synthetic spectrum. In reality, the high-frequency signals, according to Eq. (1) should damp in a more rapid way due to the refraction in the bottom.

\section{Conclusions}

Considering water column to be a compressible fluid we processed data of JAMSTEC bottom pressure sensors which were recorded during the 2003 Tokachi-Oki tsunamigenic earthquake. Spectrum analysis of bottom pressure variations proved the existence of elastic oscillations of water column. From the bottom pressure records we estimated bottom displacement amplitude $\left(\triangle H_{P G 1} \approx 0.4 \mathrm{~m}\right.$, $\left.\triangle H_{P G 2} \approx 0.15 \mathrm{~m}\right)$, duration $\left(\tau_{P G 1} \approx 8.6 \mathrm{~s}, \tau_{P G 2} \approx 7.7 \mathrm{~s}\right)$, and velocity $\left(U_{P G 1} \approx 0.093 \mathrm{~m} / \mathrm{s}, U_{P G 2} \approx 0.039 \mathrm{~m} / \mathrm{s}\right)$.

In the framework of the linear potential theory of compressible fluid we developed 3-D numerical model to describe the water layer dynamics in a real bathymetry domain. The model was used to simulate dynamical processes in the ocean during the 2003 Tokachi-oki earthquake. It appeared to be capable of reproducing the position of the main spectral maximum correctly. However, due to neglecting of crust elasticity and to lack of exact knowledge of spatiotemporal laws of bottom motion, there is an essential difference between in-situ observed and computed spectra.

In the end, we would like to note that exact simulation of dynamical processes in the tsunami source is a very com- plicated and multidisciplinary problem. It requires a plenty of high-resolution data such as bathymetry, crustal and sedimentary structure, spatiotemporal distribution of co-seismic slip and related distribution of the bottom deformation.

Acknowledgements. This work was supported by the Russian Foundation for Basic Research, projects 04-05-64297, 07-0500414 and the Federal Program for the Support of Leading Scientific Schools, project no. NSh-2104.2003.5. We are grateful to JAMSTEC and JODC for providing the data. Special thanks to $\mathrm{H}$. Matsumoto for providing bottom deformation data in a digital form.

Edited by: S. Tinti

Reviewed by: H. Matsumoto and one referee

\section{References}

Hirata, K., Aoyagi, M., Mikada, H., Kawaguchi, K., Kaiho, Y., Iwase, R., Morita, S., Fujisawa, I., Sugioka, H., Mitsuzawa, K., Suyehiro, K., Kinoshita, H., and Fujiwara, N.: Real-time geophysical measurements on the deep seafloor using submarine cable in the southern Kurile subduction zone, IEEE J. Oceanic Eng., 27(2), 170-181, 2002.

Hirata, K., Tanioka, Y., Satake, K., Yamaki, S., and Geist, E. L.: The tsunami source area of the 2003 Tokachi-oki earthquake estimated from tsunami travel times and its relationship to the 1952 Tokachi-oki earthquake, Earth Planets Space, 56, 367-372, 2004.

Kajiura, K.: Tsunami source, energy and the directivity of wave radiation, Bull. Earthq. Res. Inst. Tokyo Univ., 48, 835-869, 1970.

Kanamori, H. and Anderson, D. L., Theoretical basis of some empirical relations in seismology, Bull. Seism. Soc. Am. 65, 10731095, 1975.

Kowalik, Z., Knight, W., Logan, T., and Whitmore, P.: Numerical modeling of the global tsunami: Indonesian Tsunami of $26 \mathrm{De}-$ cember 2004, Sci. Tsunami Hazard, 23(1), 40-56, 2005.

Mikada, H., Mitsuzawa, K., Matsumoto, H., Watanabe, T., Morita, S., Otsuka, R., Sugioka, H., Baba, T., Araki, E., and Suyehiro, K., New discoveries in dynamics of an M8 earthquake Phenomena and their implications from the 2003 Tokachi-oki Earthquake using a long term monitoring cabled observatory, Tectonophysics, 426, 95-105, 2006.

Miyazaki, S., Segall, P., Fukuda, J., and Kato T.: Space time distribution of afterslip following the 2003 Tokachi-oki earthquake: Implications for variations in fault zone frictional properties, Geophys. Res. Lett., 31, L06623, doi:10.1029/2003GL019410, 2004.

Miyoshi, H.: Generation of the tsunami in compressible water (Part I), J. Oceanogr. Soc. Japan, 10, 1-9, 1954.

Nosov, M. A. and Kolesov, S. V.: Nonlinear tsunami generation mechanism in compressible ocean, Vestnik Moskovskogo Universita. Ser. 3 Fizika Astronomiya, (3), 51-54, 2005.

Nosov, M. A. and Skachko, S. N.: Nonlinear tsunami generation mechanism, Nat. Hazards Earth Syst. Sci., 1, 251-253, 2001, http://www.nat-hazards-earth-syst-sci.net/1/251/2001/.

Nosov, M. A., Kolesov, S. V., Ostroukhova, A. V., Alekseev, A. B., and Levin, B. W.: Elastic oscillations of the water layer in a tsunami source, Doklady Earth Sciences, 404(7), 1097-1100, 2005. 
Nosov, M. A.: Tsunami generation in a compressible ocean by vertical bottom motions, Izvestiya - Atmospheric and Ocean Physics, 36(5), 661-669, 2000.

Nosov, M. A.: Tsunami generation in compressible ocean, Physics and Chemistry of the Earth, Part B: Hydrology, Oceans and Atmosphere, 24(5), 437-441, 1999.

Novikova, L. E. and Ostrovsky, L. A.: On an acoustic mechanism of tsunami wave generation, Oceanology, 22(5), 693-697, 1982.

Ohmachi, T., Tsukiyama, H., and Matsumoto, H.: Simulation of tsunami induced by dynamic displacement of seabed due to seismic faulting, Bull. Seismological Soc. Am., 91(6), 1898-1909, 2001.

Okal, E. A., Alasset, P.-J., Hyvernaud, O., Schindeleì, F.: The deficient $\mathrm{T}$ waves of tsunami earthquakes, Geophys. J. Int., 152(2), 416-432, 2003.

Panza, G. F., Romanelli, F., and Yanovskaya, T. B.: Synthetic tsunami mareograms for realistic oceanic models, Geophys. J. Int., 141(2), 498-508, 2000.

Sells, C. C. L.: The effect of a sudden change of shape of the bottom of a slightly compressed ocean, Phil. Trans. Roy. Soc. London (A), 258, 495-528, 1965.
Tanioka, Y., Hirata, K., Hino, R., and Kanazawa, T.: Slip distribution of the 2003 Tokachi-oki earthquake estimated from tsunami waveform inversion, Earth Planets Space, 56, 373-376, 2004.

Titov, V. V., Rabinovich, A. B., Mofjeld, H. O., Thomson R. I., and Gonzalez, F.I.: The global reach of the 26 December 2004 Sumatra tsunami, Science, 309, 2045-2048, 2005.

Tolstoy, I. and Clay, C. S.: Ocean Acoustics - Theory and Experiment in Underwater Sound, 2nd ed., American Institute of Physics, New York, 1987.

Watanabe, T., Matsumoto, H., Sugioka, H., et al.: Offshore monitoring system records recent earthquake off Japan's northernmost island, Eos, 85(2), 14-15, 2004.

Yagi, Y.: Source rupture process of the 2003 Tokachi-oki earthquake determined by joint inversion of teleseismic body wave and strong ground motion data, Earth Planets Space, 56, 311316, 2004.

Yamanaka, Y. and Kikuchi, M.: Source process of the recurrent Tokachi-oki earthquake on September 26, 2003, inferred from teleseismic body waves, Earth Planets Space, 55, 21-24, 2003. 